04

\title{
Новые возможности зондовой регистрации анизотропных функций распределения заряженных частиц в плазме с произвольной симметрией
}

\author{
(C) В.С. Сухомлинов, ${ }^{1}$ А.С. Мустафраев, ${ }^{2}$ А.А. Страхова, ${ }^{2}$ О. Мурильо ${ }^{2}$ \\ ${ }^{1}$ Санкт-Петербургский государственный университет, фризический фракультет, \\ 198504 Санкт-Петербург, Россия \\ ${ }^{2}$ Санкт-Петербургский горный университет, \\ 199106 Санкт-Петербург, Россия \\ e-mail: v_sukhomlinov@mail.ru, alexmustafaev@yandex.ru
}

(Поступило в Редакцию 10 апреля 2017 г.)

Проведен анализ точности и систематических ошибок известного метода плоского одностороннего зонда для измерения анизотропных функций распределения электронов и ионов в плазме. В условиях, когда анизотропия обусловлена наличием электрического поля в плазме, получены аналитические соотношения, связывающие параметры плазмы и необходимое количество членов ряда. Показано, что, за исключением сильных полей, как правило, для адекватного описания функции распределения ионов, оказывается достаточно десяти членов ряда. Для сильно анизотропных функций распределения заряженных частиц предложена и апробирована методика сплайновой интерполяции экспериментальных данных, которая существенно снижает систематическую ошибку при заданном количестве членов ряда. Для часто встречающихся реальных плазменных объектов с „зеркальной симметрией“ показано, что по сравнению с наиболее общим случаем отсутствия любой симметрии при фиксированном количестве членов ряда Лежандра для функции распределения в эксперименте достигается уменьшение количества необходимых ориентаций плоского зонда до двух раз, а при одном и том же количестве ориентаций соответственно до двух раз возрастает число членов ряда, которые определяются из этих измерений.

DOI: 10.21883/JTF.2017.12.45205.2289

\section{Введение}

В работах $[1,2]$ был предложен и в дальнейшем развит авторами [3-13] зондовый метод определения анизотропных функций распределения электронов (ФРЭ) по скоростям в виде конечного ряда по полиномам Лежандра. Число членов ряда зависит от вида симметрии задачи и количества ориентаций плоского одностороннего или цилиндрического зондов, для которых регистрируются зависимости второй производной зондового тока от потенциала зонда.

Аналогичный метод применительно к функции распределения ионов (ФРИ) с использованием плоского одностороннего зонда был предложен в работах [14-16]. Основная идея метода применительно к ионам состоит в использовании той части зондовой характеристики, для которой ионы движутся около зонда в задерживающем поле, а электроны - в ускоряющем. Поскольку известно, что электронный ток на плоский зонд в этих условиях не зависит от потенциала зонда $[17,18]$, вторая производная зондового тока несет информацию о ФРИ по скоростям. Техника регистрации и обработка результатов измерений производятся аналогично тому, как это делается при определении ФРЭ методом плоского одностороннего зонда [13].

Суть метода определения анизотропной функции распределения (ФР) из результатов зондовых измерений состоит в следующем. Как известно, при выполнении ряда условий в плазме $[1,2,14]$ вторая производная зондового тока $I^{\prime \prime}(e U, \alpha)$ по потенциалу зонда $(e, \alpha-$ заряд электрона и угол между внешней нормалью к проводящей поверхности зонда и выделенным направлением соответственно) и ФР $f_{\varepsilon}(E, \theta)$ (где $\theta-$ угол между осью симметрии и направлением движения заряженной частицы, имеющей энергию $E$ ) связаны соотношением

$I^{\prime \prime}(e U, \alpha)=A\left[f_{\varepsilon}(e U, \alpha)-\frac{1}{2 \pi} \int_{0}^{2 \pi} d \varphi^{\prime} \int_{e U}^{\infty} \frac{\partial f_{\varepsilon}\left(E, \theta^{\prime}\right)}{\partial e U} d E\right]$,

где $\cos \theta^{\prime}=\sqrt{\frac{e U}{\varepsilon}} \cos \alpha+\sqrt{1-\frac{e U}{\varepsilon}} \sin \alpha \cos \varphi^{\prime}, A=2 \pi \times$ $\times \frac{S_{p} e^{3}}{m^{2}}, S_{p}, m, e-$ площадь зонда, масса заряженной частицы (электрона или иона) и ее заряд, соответственно; $\varphi^{\prime}-$ разность азимутальных углов скорости частицы и внешней нормали к поверхности зонда в выбранной системе координат, $E$ - энергия заряженной частицы. Для определения $f_{\varphi} E, \theta$ сначала измеряется зависимость второй производной зондового тока по потенциалу зонда $U$ при различных углах $\alpha$. Затем вторая производная представляется в виде конечного ряда по полиномам Лежандра, число членов которого, например, для осесимметричного случая равно числу ориентаций зонда $N[1,2]$

$$
I^{\prime \prime}\left(e U, \alpha_{i}\right)=A \sum_{k=0}^{N} D_{k}(e U) L_{k}\left(\cos \alpha_{i}\right)
$$


(а в случае отсутствия симметрии $\left.-N^{2}[13]\right)$. При этом ФР также представляется в виде аналогичного ряда

$$
f_{\varepsilon}(E, \theta)=\sum_{k=0}^{N} C_{k}(E) L_{k}(\mu)
$$

где $\mu=\cos \theta$. Затем, используя экспериментальные данные и (в осесимметричном случае) разложение (1), находят коэффициенты разложения второй производной зондового тока в ряд по полиномам Лежандра $D_{k}(e U)$. Наконец, из этих коэффициентов вычисляются коэффициенты в аналогичном разложении $\Phi$ Р $C_{k}(e U)$ с использованием известной связи [13]

$$
C_{k}(e U)=D_{k}(e U)+\left.\frac{1}{2 e U} \int_{e U}^{\infty} D_{k}(\varepsilon) \frac{d L_{k}(x)}{d x}\right|_{x=\sqrt{\frac{\varepsilon}{e U}}} d \varepsilon .
$$

При отсутствии симметрии в плазме в наиболее общем случае аналоги разложений (2), (3) имеют более сложный вид (этот случай мы исследуем ниже, см. формулу (18)) [13].

При определении ФР данным методом априори вносятся следующие систематические ошибки:

- ошибка из-за недостаточного количества полиномов Лежандра для описания ФР с большой анизотропией;

- ошибка в определении коэффициентов Лежандра второй производной зондового тока (из-за обрезания ряда по полиномам Лежандра), приводящая к ошибке определения коэффициентов Лежандра для ФР.

Одним из наиболее существенных недостатков данного метода определения ФРЭ и ФРИ, на наш взгляд, является то, что заранее не ясно, сколько членов ряда необходимо учитывать, чтобы ФР, определенная в виде конечного ряда, адекватно описывала реальную функцию. Интуитивно понятно, что чем больше анизотропия $\Phi Р$, тем больше членов ряда потребуется. Так, если ФР изотропна, то достаточно одного члена $(N=0)$, а если все частицы движутся в одном направлении, то потребуется бесконечное число членов. С экспериментальной точки зрения знание числа членов этого ряда весьма существенно, поскольку от него зависит угловое разрешение, с которым нужно проводить измерения, что определяет конструкцию основных узлов экспериментальной установки. Очевидно, что случаи, когда достаточно двух членов и когда необходимо более десяти, кардинально различаются по своей аппаратурной реализации.

В работе исследуется несколько возможных способов уменьшения систематических ошибок метода плоского одностороннего зонда при измерении анизотропных ФР как для электронов, так и для ионов.

\section{Зависимость необходимого количества ориентаций зонда от параметров плазмы при измерении функции распределения ионов}

Рассмотрим ситуацию, когда ФРИ имеет осевую симметрию. В наиболее общем случае анизотропия может быть вызвана также наличием в плазме поверхностей, поглощающих заряженные частицы, не изотропных источников этих частиц с различными распределениями по энергиям и т. п. Исследуем ФРИ в собственном газе и рассмотрим ситуацию, когда единственная причина анизотропии - наличие электрического поля в плазме. Основные процессы, которые реализуются с участием ионов в низкотемпературной газоразрядной плазме при низких и средних давлениях и, в известном смысле, небольших плотностях заряженных частиц, которые могут оказать влияние на формирование ФРИ, следующие:

- перезарядка на нейтральных частицах;

- упругое рассеяние на нейтральных частицах;

- процесс конверсии атомных ионов в молекулярные;

- при больших концентрациях заряженных частиц процесс тройной рекомбинации атомарных ионов;

- диссоциативная рекомбинация молекулярных ионов.

Рассмотрим возможное влияние на ФРИ вышеперечисленных процессов. Как известно, процесс перезарядки иона (особенно на собственном атоме) является одним из основных в газоразрядной плазме. В процессе движения между столкновениями с нейтралами ион увеличивает свою энергию, ускоряясь в электрическом поле и, таким образом, ФРИ приобретает анизотропию. При упругих столкновениях часть энергии иона, которую он приобрел, двигаясь между столкновениями, перераспределяется в плоскость, ортогональную направлению поля, и, таким образом, ФРИ становится менее анизотропной. При повышенных давлениях возможна так называемая конверсия атомарных ионов при тройных столкновениях с образованием молекулярных ионов. При этом константа скорости этого процесса практически не зависит от относительной энергии сталкивающихся частиц [19]. Отсюда следует, что учет конверсии для ФР атомарных ионов практически не влияет на вид угловой и энергетической зависимостей ФРИ. Константа скорости тройной рекомбинации атомарных ионов, которая оказывает влияние на скорость их нейтрализации при повышенных плотностях тока, сильно зависит от средней энергии электронов $\left(\sim \frac{1}{\bar{E}_{e}^{4.5}}, \bar{E}_{e}-\right.$ средняя энергия электронов $)$ и практически не зависит от энергии иона [19]. Отсюда следует, что этот процесс также не влияет на формирование ФРИ.

В силу сравнительно слабой зависимости сечения диссоциативной рекомбинации молекулярных ионов от энергии относительного движения иона и атома [19] этот процесс также будет слабо влиять на ФР молекулярных ионов. 
Таким образом, можно сделать вывод, что ФРИ, рассчитанная с учетом только перезарядки, является максимально анизотропной из всех возможных. Учитывая вышесказанное, можно утверждать, что число членов ряда (3), необходимое для адекватного описания ФРИ, которая соответствует процессу резонансной перезарядки и не учитывает других процессов, является для числа $N$ необходимых членов ряда по полиномам Лежандра при любых условиях в плазме оценкой сверху. Напомним, что мы имеем в виду ситуацию, когда единственной причиной анизотропии ФРИ является электрическое поле в плазме.

В работах $[14,16]$ было получено выражение для ФРИ при учете только перезарядки. Было выяснено, что ФРИ зависит от двух параметров $\varepsilon_{0}=\frac{k_{0} T_{a}}{E_{0} \lambda_{e x}}, T_{a}$, где $E_{0}$ - электрическое поле, $\lambda_{e x}$ - длина пробега иона относительно процесса перезарядки, $T_{a}$ - температура нейтральных частиц, $k_{0}$ - постоянная Больцмана. Сравнение расчетов ФРИ по полученным соотношениям с экспериментально восстановленными методом плоского одностороннего зонда показали, что, при любых значениях параметра $\varepsilon_{0}$, количестве определенных коэффициентов $N$ в разложении ФРИ в ряд по полиномам Лежандра и заданной величине $\delta$ существует такое значение энергии иона $\varepsilon_{\max }\left(N, \varepsilon_{0}, T_{a}, \mu\right)$, что для всех энергий иона $\varepsilon<\varepsilon_{\max }\left(N, \varepsilon_{0}, T_{a}, \mu\right)$ выполняется неравенство

$$
\Delta(E, \mu)=\frac{\left|R_{N}(E, \mu)\right|}{\bar{f}_{\varepsilon}(E, \mu)}<\delta,
$$

где $\mu=\cos \theta, \bar{f}_{\varepsilon}(E, \mu)=f_{\varepsilon}(E, \theta)-\Phi Р$ по энергиям, $R_{N}(E, \mu)$ - остаток ряда при аппроксимации ФР конечным рядом по полиномам Лежандра с точными коэффициентами:

$$
\begin{gathered}
R_{N}\left(E, \mu=\sum_{k=n}^{\infty} C_{k}(E) L_{k}(\mu),\right. \\
C_{k}(E)=(k+0.5) \int_{-1}^{1} \bar{f}_{\varepsilon}(E, \mu) L_{k}(\mu) d \mu .
\end{gathered}
$$

При этом максимум величины $\Delta(E, \mu)$ при любых значениях энергии иона $E$ достигается при $\mu=1$, т.е. в направлении поля. Тогда можно, полагая (с учетом оцененной авторами [14,16] ошибки измерения ФРИ в $10 \%) \delta=0.1$ и используя найденную в этих работах ФРИ с учетом только резонансной перезарядки, для любых $N, \varepsilon_{0}$ найти $\varepsilon_{\max }\left(N, \varepsilon_{0}, T_{a}, 1\right)>\varepsilon_{\max }\left(N, \varepsilon_{0}, T_{a}, \mu\right)$, которая и будет искомой оценкой сверху. В этом случае при любом $\mu$ и заданных $N, \varepsilon_{0}$ разница между реальной и восстановленной ФРИ не будет превышать 10\%. В Приложении 1 приведены формулы для функции $\varepsilon_{\max }\left(N, \varepsilon_{0}, 1\right)$ в диапазонах параметров $0.01 \leq \varepsilon_{0} \leq 1.5 ; 300 \mathrm{~K} \leq T_{a} \leq 900 \mathrm{~K} ; a \leq N \leq 10$, полученные в $[14,16]$. Отметим, что расчеты с использованием зависимости сечения перезарядки от относительной энергии иона и атома для различных пар ион-атом показали, что учет этой зависимости слабо (в пределах 10\%) увеличивает рассчитанную величину $\varepsilon_{\max }\left(N, \varepsilon_{0}, T_{a}, 1\right.$.

\section{Применение теории сплайнов для уменьшения в эксперименте числа ориентаций зонда при сохранении точности определения ФР в газоразрядной плазме с зеркальной
симметрией}

Рассмотрим некоторые возможности уменьшения систематических погрешностей зондовой регистрации анизотропных ФР заряженных частиц в виде конечного ряда по полиномам Лежандра. По-прежнему будем рассматривать осесимметричный случай.

По аналогии с (6) определим остаток ряда $R_{N I}(e U, \alpha)$ при аппроксимации второй производной зондового тока $I^{\prime \prime}(e U, \alpha)$ конечным рядом с точными коэффициентами $D_{k}(e U)$ по формуле

$$
\begin{gathered}
R_{N I}(e U, \alpha)=A \sum_{k=N}^{\infty} D_{k}(e U) L_{k}(\cos \alpha), \\
D_{k}(e U)=(k+0.5) \int_{-1}^{1} I^{\prime \prime}(e U, \alpha) L_{k}(\cos \alpha) d \cos \alpha .
\end{gathered}
$$

Пользуясь определением $L_{k}(\mu)$ [20] и интегрируя по частям соотношение (6) $k$ раз, получаем

$$
R_{N}(E, \mu)=\sum_{k=N}^{\infty} \frac{(k+0.5)}{2^{k} k !} L_{k}(\mu) \int_{-1}^{1} \bar{f}_{\varepsilon}(E, \mu)^{(k)}\left(1-\mu^{2}\right)^{k} d \mu,
$$

где $\bar{f}_{\varepsilon}(E, \mu)^{(k)}=\frac{\partial^{k} \bar{f}_{\varepsilon}(E, \mu)}{\partial \mu^{k}}$. Из $(7)$, учитывая, что

$$
\int_{-1}^{1}\left(1-\mu^{2}\right)^{k} d \mu=B\left(k+1, \frac{1}{2}\right)=\frac{\Gamma(k+1) \Gamma\left(\frac{1}{2}\right)}{\Gamma\left(k+\frac{3}{2}\right)},
$$

где $B(x, y), \Gamma(x)$ - бета- и гамма-функции соответственно [20], имеем оценку

$$
R_{N}(E, \mu) \leq \sum_{k=N}^{\infty} \frac{2^{k} k !}{2 k !}\left|\bar{f}_{\varepsilon}(E, \mu)^{(k)}\right|_{\max }\left|L_{k}(\mu)\right|,
$$

где $\left|\bar{f}_{\varepsilon}(E, \mu)^{(k)}\right|_{\max }$ - максимальное значение модуля производной ФР порядка $k$ на промежутке $-1 \leq \mu \leq 1$ при заданной энергии $E$. При $N \gg 1$, в случае сходимости ряда (7) и при отсутствии острого максимума у функции $\bar{f}_{\varepsilon}(E, \mu)^{(k)}$ на промежутке $-1 \leq \mu \leq 1$ в точке, не совпадающей с $\mu=0$, пользуясь методом перевала [21], можно получить более точную оценку

$$
R_{N}(E, \mu) \leq \sqrt{\pi} \sum_{k=N}^{\infty} N \frac{\sqrt{k}}{2^{k} k !} \bar{f}_{\varepsilon}(E, \mu)_{\mu=0}^{(k)} L_{k}(\mu) .
$$


При $\mu=0$ из (8) имеем, что нечетные члены ряда равны 0 , а четные члены образуют знакопеременный ряд, откуда получаем при четных $N \gg 1$ :

$$
\begin{gathered}
\left|R_{N}(E, 0)\right| \leq \frac{(\sqrt{2})^{N}(N-1) N !}{2 N !}\left|\bar{f}_{\varepsilon}(E, \mu)^{(N)}\right|_{\max } \\
\cong \frac{\exp (-0.04 N)}{\sqrt{2} N^{N-1}}\left|\bar{f}_{\varepsilon}(E, \mu)^{(N)}\right|_{\max } \\
R_{N+1}(E, 0)=R_{N}(E, 0)
\end{gathered}
$$

При выводе этой формулы использовалась формула Стирлинга для $N$ !, справедливая при $N \rightarrow \infty$. Совершенно аналогичные формулам (7)-(10) соотношения можно получить для $R_{N I}(e U, \alpha)$ с заменой в них $\bar{f}_{\varepsilon}(E, \mu)^{(k)}$ на $I^{\prime \prime}(e U, \alpha)_{\cos \alpha}^{(k)}$ (отметим, что производная здесь берется не по углу $\alpha$, а по его косинусу) и $D_{k}(e U)$ на $C_{k}(E)$.

Полученные оценки для погрешности представления ФР в виде конечного ряда по полиномам Лежандра, очевидно, применимы как к ФРИ, так и к ФРЭ, обладающим произвольной степенью анизотропии. Из полученных выше соотношений видно, что, действительно, чем больше значение максимума производной функции по аргументу $\mu$, тем больше остаток ряда при фиксированном значении $N$ и тем больше требуется членов ряда для адекватного описания конечным рядом функции $\bar{f}_{\varepsilon}(E, \mu)$. Две очевидных предельных ситуации - это сферически симметричная $\bar{f}_{\varepsilon}(E, \mu)$, когда все $\bar{f}_{\varepsilon}(E, \mu)^{(k)}=0$ при $k>0$, и случай, когда $\bar{f}_{\varepsilon}(E, \mu) \propto \delta\left(\mu-\mu_{0}\right)$, где $\delta\left(\mu-\mu_{0}\right)-$ дельта-функция Дирака. В этом случае величина $\left|\bar{f}_{\varepsilon}(E, \mu)^{(k)}\right|_{\max }$ не ограничена и ряд $R_{N}(E, \mu)$ расходится при любом значении $N$.

Рассмотрим, как влияет на погрешность определения ФР аппроксимация конечным рядом второй производной зондового тока. Как уже говорилось, суть зондового метода определения ФР заключается в измерении второй производной зондового тока по потенциалу зонда относительно плазмы при $N$ различных ориентациях зонда в необходимом диапазоне потенциала зонда относительно плазмы и нахождению по этим измерениям $N$ первых коэффициентов Лежандра $D_{k}(e U)$. Затем с помощью соотношения (4) вычисляются соответствующие коэффициенты Лежандра $C_{k}(e U)$ ФР. При этом, как мы говорили, представление $I^{\prime \prime}(e U, \alpha)$ в виде конечного ряда приводит к погрешностям в определении коэффициентов $D_{k}(e U)$, а значит, и $C_{k}(e U)$. Это очевидно, поскольку в равенстве (2) с суммой в правой части до максимального $k_{\max }=N$ слева стоит точное экспериментально найденное значение второй производной $I^{\prime \prime}(e U, \alpha)$, которое является суммой бесконечного числа членов, и, таким образом, само равенство с $k_{\max }=N$ является приближенным и удовлетворяется с коэффициентами $D_{k}^{N}(e U)=D_{k}(e U)+\Delta D_{k}^{N}(e U)$, отличными от $D_{k}(e U)$ при разложении в бесконечный ряд Лежандра. То есть с учетом вышесказанного, и принимая во внимание уравнение (4), можно записать

$$
\begin{gathered}
\Delta C_{k}^{N}(e U)=\Delta D_{k}^{N}(e U)+\left.\frac{1}{2 e U} \int_{e U}^{\infty} \Delta D_{k}^{N}(\varepsilon) \frac{d L_{k}(x)}{d x}\right|_{x=\frac{\varepsilon}{e U}} d \varepsilon, \\
C_{k}^{N}(e U)=D_{k}^{N}(e U)+\left.\frac{1}{2 e U} \int_{e U}^{\infty} D_{k}^{N}(\varepsilon) \frac{d L_{k}(x)}{d x}\right|_{x=\frac{\varepsilon}{e U}} d \varepsilon
\end{gathered}
$$

где $\Delta D_{k}^{N}(e U), \Delta C_{k}^{N}(e U)-$ систематические ошибки (зависящие от числа членов $N$ ) в определении коэффициентов $D_{k}(e U)$ и $C_{k}(e U)$, соответственно. С учетом (6а) имеем

$$
\begin{gathered}
A \sum_{k=0}^{N} \Delta D_{k}^{N}(e U) L_{k}\left(\cos \alpha_{i}\right)=R_{N I}\left(e U, \alpha_{i}\right), \quad i=0 \ldots N \\
A \sum_{k=0}^{N} D_{k}^{N}(e U) L_{k}\left(\cos \alpha_{i}\right)=I^{\prime \prime}\left(e U, \alpha_{i}\right) .
\end{gathered}
$$

Соотношения (12), (12а) можно рассматривать, как системы $N$ уравнений для нахождения величин $\Delta D_{k}^{N}(e U)$, $D_{k}^{N}(e U), k=0 \ldots N$. Определитель этих систем при любых $N, \alpha_{i}$ отличен от нуля, и поэтому при любых $N, \alpha_{i}$ у нее существует ненулевое решение. По этой же причине, поскольку $R_{N I}\left(e U, \alpha_{i}\right)_{N \rightarrow \infty} \rightarrow 0$ при любых $e U, \alpha_{i}$, $\Delta D_{k}^{N}(e U)_{N \rightarrow \infty} \rightarrow 0$. Используя соотношение (11), получаем связь между величинами второй производной зондового тока и экспериментально определенной ФР $\bar{f}_{\varepsilon e x}\left(E, \cos \alpha_{i}\right)$ :

$$
\begin{aligned}
A \bar{f}_{\varepsilon e x}\left(E, \cos \alpha_{i}\right)= & I^{\prime \prime}\left(E, \alpha_{i}\right)+\frac{A}{2 e U} \sum_{k=0}^{N} L_{k}\left(\cos \alpha_{i}\right) \\
& \times\left.\int_{e U}^{\infty} D_{k}^{N}(\varepsilon) \frac{d L_{k}(x)}{d x}\right|_{x=\frac{\varepsilon}{e U}} d \varepsilon,
\end{aligned}
$$

где $D_{k}^{N}(e U)$ находятся из системы уравнений (12а). Таким образом мы видим, что погрешность определения ФР зависит не только от числа членов ряда $N$, но и от выбора углов $\alpha_{i}, i=0 \ldots N$, поскольку этот выбор определяет матрицу системы (12). Принимая во внимание соотношение (4), из формулы (13) получаем

$$
\begin{aligned}
& \Delta \bar{f}_{\varepsilon e x}\left(E, \cos \alpha_{i}\right) \frac{1}{2 e U}\left\{\sum_{k=0}^{N} L_{k}\left(\cos \alpha_{i}\right)\right. \\
& \times\left.\int_{e U}^{\infty} \Delta D_{k}^{N}(\varepsilon) \frac{d L_{k}(x)}{d x}\right|_{x=\frac{\varepsilon}{e U}} d \varepsilon \\
& \left.-\left.\sum_{k=N}^{\infty} L_{k}\left(\cos \alpha_{i}\right) \int_{e U}^{\infty} D_{k}^{N}(\varepsilon) \frac{d L_{k}(x)}{d x}\right|_{x=\frac{\varepsilon}{e U}} d \varepsilon\right\},
\end{aligned}
$$

где $\Delta \bar{f}_{\varepsilon e x}(E, \cos \alpha)=\bar{f}_{\varepsilon e x}(E, \cos \alpha)-\bar{f}_{\varepsilon e x}(E, \cos \alpha)$. 
Таким образом, мы видим, что существует систематическая ошибка определения угловой зависимости ФР при представлении второй производной в виде конечного ряда по полиномам Лежандра даже для углов $\alpha=\alpha_{i}$, при которых вторая производная определяется точно (т.е. без систематической ошибки). Эта ошибка для ФР складывается из двух слагаемых. Первое связано с не точным определением коэффициентов $D_{k}(\varepsilon)$, второе - с тем, что представление в виде конечного ряда неверно описывает угловую зависимость второй производной зондового тока. Обе ошибки, очевидно, стремятся к нулю при $N \rightarrow \infty$.

В принципе возможен альтернативный способ решения поставленной задачи. А именно можно, используя $N$ измерений зондового тока, аппроксимировать зависимость от угла $I^{\prime \prime}(e U, \alpha)$ при разных значениях потенциала $U$ сплайнами (например, третьего порядка) и затем вычислять произвольное (необходимое для точного описания результатов интерполяции) количество коэффициентов Лежандра для второй производной, используя полученные гладкие кривые. Роль аналога остатка ряда по полиномам Лежандра $R_{N I}(e U, \alpha)$ для величины $I^{\prime \prime}(e U, \alpha)$ здесь будет играть ошибка интерполяции сплайнами $S_{N I}(e U, \alpha)$. Если измерения проводятся при равноотстоящих значениях углов $\alpha_{i}$ с постоянным шагом $\Delta \alpha_{i}=\frac{\pi}{N}$, то, как известно, для абсолютной ошибки интерполяции кубическими сплайнами верна оценка [22]

$$
\left|S_{N I}(e U, \alpha)\right| \leq \frac{5}{384} \Delta \alpha_{i}^{4}\left|I^{\prime \prime}(e U, \alpha)_{\alpha}^{(4)}\right| \cong \frac{1.3}{n^{4}}\left|I^{\prime \prime}(e U, \alpha)_{\alpha}^{(4)}\right|
$$

где $I^{\prime \prime}(e U, \alpha)_{\alpha}^{(4)}$ - четвертая производная величины $I^{\prime \prime}(e U, \alpha)$ по углу $\alpha$. При интерполяции второй производной тока $I^{\prime \prime}(e U, \alpha)$ кубическими сплайнами по результатам $N$ измерений и приемлемой (для конкретной задачи) относительной ошибке этой интерполяции $\frac{\left|S_{N I}(e U, \alpha)\right|}{I^{\prime \prime}(e U, \alpha)}$ можно, таким образом, найти любое число $M>N$ коэффициентов Лежандра $D_{k}^{M}(e U)$, а значит, и $C_{k}^{M}(e U)$. При этом, поскольку при возрастании $M$ уменьшается остаток $R_{M I}(\varepsilon, \mu)$ и ошибка $\Delta D_{k}^{M}(e U)$, $k==\ldots M$, можно, увеличивая число $M$, добиться того, чтобы выполнялось неравенство

$$
\left|S_{N I}(e U, \alpha)\right| \approx\left|R_{M I}(\varepsilon, \cos \alpha)\right|
$$

Описанная процедура будет, очевидно, иметь смысл при выполнении $\left|S_{N I}(e U, \alpha)\right|<\left|R_{N I}(\varepsilon, \cos \alpha)\right|$, при котором с учетом (14a) имеем $\left|R_{M I}(\varepsilon, \cos \alpha)\right|<\left|R_{N I}(\varepsilon, \cos \alpha)\right|$.

Если же выполняется обратное неравенство, то применение числа членов разложения второй производной $M>N$ не приведет к уменьшению ошибки определения ФР. Иными словами, измеряя $I^{\prime \prime}(e U, \alpha)$ при $N$ значениях угла $\alpha$, можно найти ФРИ в виде разложения по $M>N$ полиномам Лежандра.

При высокой степени анизотропии ФР, когда число ориентаций зонда слишком мало́ для описания второй производной зондового тока $I^{\prime \prime}(e U, \alpha)$ и ФР в виде конечного ряда по полиномам Лежандра, применение сплайнов для вычисления членов этого ряда с большим, чем число ориентаций зонда номером, существенно снижает ошибку определения ФР.

Наконец, обсудим часто встречающийся в практике экспериментальных исследований случай так называемой зеркальной симметрии. Сначала предположим, что в плазме отсутствует какой-либо тип симметрии. Тогда предлагается представить $Ф \mathrm{P} f_{\varepsilon(R, \theta, \varphi)}$ в виде разложения по присоединенным функциям Лежандра 1-го рода $P_{l}^{|m|}[13]$

$$
\begin{gathered}
f_{\varepsilon(R, \theta, \varphi)}=\sum_{l=0}^{\infty} \sum_{m=-l}^{l} f_{l m}(E) Y_{l m}(\theta, \varphi), \\
Y_{l m}(\theta, \varphi)=\frac{1}{2} \sqrt{\frac{(2 l+1)(l-|m|) !}{\pi(l+|m|) !}} P_{l}^{|m|}(\cos \theta) e^{i m \varphi}, \\
m=-l \ldots l,
\end{gathered}
$$

где $Y_{l m}(\theta, \varphi)$ - сферические функции. Здесь $f_{l m}(v)-$ комплексные величины, которые подлежат определению из измерений зависимости второй производной зондового тока $I^{\prime \prime}\left(e U, \alpha, \varphi_{0}\right)$ от потенциала зонда $U$ при различных его ориентациях

$$
I^{\prime \prime}\left(e U, \alpha, \varphi_{0}\right)=A \sum_{l=0}^{\infty} \sum_{m=-l}^{l} D_{l m}(e U) Y_{l m}\left(\alpha, \varphi_{0}\right),
$$

где $\varphi-$ азимутальный угол скорости заряженной частицы в выбранной лабораторной системе координат $X Y Z$; $\alpha, \varphi_{0}-$ полярный и азимутальный углы соответственно внешней нормали к проводящей поверхности к зонду в этой системе координат. При этом коэффициенты рядов в формулах (15) и (16) связаны интегральным уравнением, аналогичным (4)

$$
f_{l m}(e U)=D_{l m}(e U)+\left.\frac{1}{2 e U_{p}} \int_{e U}^{\infty} D_{l m}(\varepsilon) \frac{d L_{l}(x)}{d x}\right|_{x=\frac{\varepsilon}{e U}} d \varepsilon .
$$

Таким образом, при отсутствии симметрии процесс определения ФР сводится к следующему. Ограничиваясь во внешней сумме соотношения (16) $N$ членами, при $N^{2}$ ориентациях зонда производятся измерения зависимости второй производной зондового тока от потенциала зонда. Используя эти данные, из системы $N^{2}$ линейных алгебраических уравнений находим такое же количество коэффициентов $D_{l m}(e U)$ и с помощью соотношения (17) вычисляем $N^{2}$ коэффициентов $f_{l m}(e U)$. Это дает нам ФР $f_{\varepsilon}(R, \theta, \varphi)$ в виде ряда (15) до $l=N-1$ (т.е. тоже содержащим во внешней сумме $N$ членов).

Рассмотрим теперь плазму газового разряда, которая обладает тем свойством, что в ней существует плоскость, относительно которой свойства плазмы обладают симметрией. Будем называть такую симметрию 
зеркальной. Тогда, выбирая систему координат $X Y Z$ так, что плоскость $Z X$ совпадает с плоскостью симметрии, получим, что ФР четна по азимутальному углу $\varphi$. Такая ситуация реализуется на практике весьма часто, например, когда зондовые измерения выполняются в цилиндрическом разряде во внеосевой (в том числе и пристеночной) области. В этом случае соотношение (15) приобретает вид

$$
\begin{gathered}
f_{\varepsilon}(R, \theta, \varphi)=\sum_{l=0}^{\infty} \sum_{m=0}^{l} f_{l m}(E) y(\theta, \varphi), \\
y_{l m}(\theta, \varphi)=\sqrt{\frac{(2 l+1)(l-|m|) !}{\pi(l+|m|) !}} P_{l}^{|m|}(\cos \theta) \cos (m \varphi), \\
m=0 \ldots l .
\end{gathered}
$$

Аналогично преобразуется и выражение (16) для $I^{\prime \prime}\left(e U, \alpha, \varphi_{0}\right)$. Нетрудно видеть, что в случае зеркальной симметрии в плазме коэффициенты $f_{l m}(E)$ действительны и, таким образом, при представлении ФР в виде конечного ряда, содержащим $N$ функций Лежандра, необходимо определить всего $0.5 N(N+1)$ коэффициентов.

Таким образом, удается значительно уменьшить количество необходимых ориентаций плоского зонда для определения ФР заряженных частиц с заданной систематической ошибкой для часто встречающейся в плазменных исследованиях зеркальной симметрии.

Для иллюстрации вышеприведенных соотношений мы будем проводить расчеты для модельной ФР, которая определена следующими формулами:

$$
\begin{gathered}
f_{\varepsilon m}(E, \theta)=T(E) \psi(\mu) ; \quad T(E)=B \exp \left[-\frac{\left(E-E_{0}\right)^{2}}{\Delta_{T} E^{2}}\right] ; \\
B=\frac{2}{\sqrt{\pi} \Delta_{T} E\left[1+\operatorname{erf}\left(\frac{E_{0}}{\Delta_{T} E}\right)\right]} \\
\psi(\mu)=B \exp \left(\frac{\mu-1}{\beta}\right) ; \quad B=\frac{1}{2 \pi \beta\left[1-\exp \left(-\frac{2}{\beta}\right)\right]}
\end{gathered}
$$

где $\Delta_{T} E, E_{0}, \beta$ - параметры ФР, которые определяют ее ширину по энергиям, наиболее вероятную энергию и угловую анизотропию соответственно. Функция $T(E)$ описывает гауссовское распределение по энергиям, а $\psi(\mu)$ при малых параметрах $\beta-$ ускоренный в электрическом поле пучок заряженных частиц, который до ускорения имел максвелловское изотропное по полусфере (в сторону ускорения) распределение, при этом $\beta=\frac{k_{0} T_{0}}{2 e U_{0}}$ $\left(T_{0}, U_{0}\right)$ - температура максвелловского распределения до ускорения в поле и разность потенциалов в ускоряющем поле соответственно). Обе функции использовались для моделирования низковольтного пучкового разряда в гелии в [23], а $\psi(\mu)$ - также и в [24,25].

\section{Обсуждение полученных результатов}

На рис. 1 и 2 для примера приведены зависимости $\varepsilon_{\max }\left(N, \varepsilon_{0}, T_{a}, 1\right)$ от параметра $\varepsilon_{0}$ в диапазоне $0.2 \leq$ $\leq \varepsilon_{0} \leq 0.7$ при различных $N$ и $T_{a}$ соответственно. Видно, что с увеличением $\varepsilon_{0}, N, T_{a}$, величина $\varepsilon_{\max }\left(N, \varepsilon_{0}, T_{a}, 1\right)$ растет. Причины ее роста с $\varepsilon_{0}, N$, вполне очевидны.

При увеличении температуры атомов (при сохранении постоянным параметра $\varepsilon_{0}$ ) максимум ФРИ ионов смещается в сторону больших энергий $[14,16]$. Соответственно в эту же сторону смещается диапазон энергий, где распределение ионов по направлениям движения изотропно (как отмечалась в $[14,16]$, в результате перезарядки ионы рождаются с изотропным распределением и далее за счет ускорения в поле на длине пробега их угловое распределение приобретает анизотропию). При смещении области изотропии в сторону больших энергий

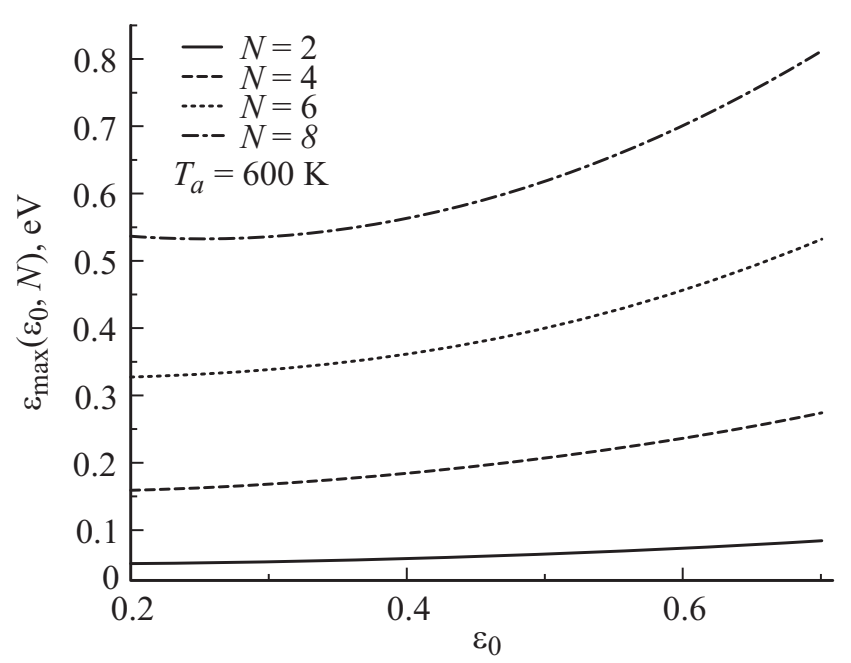

Рис. 1. Зависимость величины $\varepsilon_{\max }\left(N, \varepsilon_{0}, T_{a} 1\right)$ от параметра $\varepsilon_{0}$ в диапазоне значений от 0.2 до 0.7 при различных $N$; температура атомов $T_{a}=600 \mathrm{~K}$.

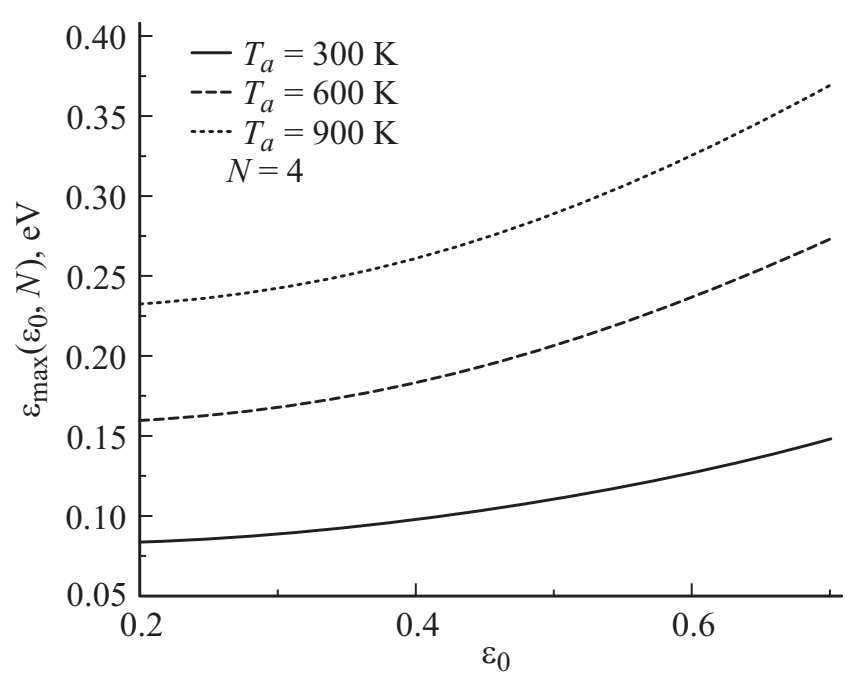

Рис. 2. Зависимость величины $\varepsilon_{\max }\left(N, \varepsilon_{0}, T_{a} 1\right)$ от параметра $\varepsilon_{0}$ в диапазоне значений от 0.2 до 0.7 при различных $T_{a} ; N=4$. 
для приобретения ФРИ анизотропии данной величины необходима, очевидно, большая энергия, полученная за счет поля. Но это и означает увеличение $\varepsilon_{\max }\left(N, \varepsilon_{0}, T_{a} 1\right)$ при росте температуры $T_{a}$.

Мы не приводим формулы для $\varepsilon_{0} \geq 1.5$ и $\varepsilon_{0} \leq$ $\leq 0.01$, по следующим причинам. Расчеты дают, что $\varepsilon_{\max }(3,1.5,300,1)=2 \mathrm{eV} ; \quad \varepsilon_{\max }(3,1.5,900,1)=3.2 \mathrm{eV}$. В соответствии с вышесказанным при $N>3$ и $\varepsilon_{0}>$ $>1.5$ эта величина будет еще больше. Как показывают оценки, при $\varepsilon_{0}=1.5$ и энергиях ионов более $2-3 \mathrm{eV}$ ФРИ настолько мала, что ее определение методом одностороннего плоского зонда затруднительно [14-16]. Таким образом, можно заключить, что в рассматриваемом случае анизотропии ФРИ из-за электрического поля для полного ее восстановления при $\varepsilon_{0}>1.5$ достаточно трех-четырех членов ряда (в зависимости от диапазона энергий) по полиномам Лежандра.

Что касается диапазона $\varepsilon_{0} \leq 0.01$, то такое значение этого параметра соответствует весьма высокой величине электрического поля (например, для случая $\mathrm{Ar}^{+}$в $\mathrm{Ar}-$ $\frac{E}{P} \cong 420 \frac{\mathrm{V}}{\mathrm{cm} \text { Torr }}$, а для $\mathrm{Hg}^{+}$в $\left.\mathrm{Hg}-\frac{E}{P} \cong 1000 \frac{\mathrm{V}}{\mathrm{cm} \text { Torr }}\right)$ и ФРИ имеет высокую степень анизотропии. При дальнейшем уменьшении параметра $\varepsilon_{0}$ количество членов ряда по полиномам Лежандра, необходимое для описания ФРИ в значимом диапазоне энергий ионов, становится слишком велико для реализации метода измерения ФРИ с помощью плоского одностороннего зонда, а $\varepsilon_{\max }\left(N, \varepsilon_{0}, T_{a} 1\right)$ становится слабо меняющейся функцией параметров $N, \varepsilon_{0}$. В пределе $\varepsilon_{0} \rightarrow 0$ условия, при которых угловая зависимость ФРИ представима в виде ряда по полной ортогональной системе функций, перестают соблюдаться. В настоящей работе мы ограничились числом $N \leq 10$, поскольку бо́льшие значения этого параметра сложно реализуемы в эксперименте при определении ФРИ, случай же $N=0$ нами также не рассматривался, поскольку в этой ситуации, очевидно, $\varepsilon_{\max }\left(N, \varepsilon_{0}, T_{a} 1\right) \sim k T_{a}$.

Отметим, что для случаев, рассмотренных в $[14,15]$, расчеты по формулам, приведенным в Приложении 1, дают согласующиеся с экспериментом результаты. Так, для случая $\mathrm{Hg}^{+}$в $\mathrm{Hg}$ при $T_{a}=410 \mathrm{~K}, \varepsilon_{0}=0.025, N=6$ получаем $\varepsilon_{\max }=0.2 \mathrm{eV}$, что полностью соответствует данным [14]; для $\mathrm{He}^{+}$в Не при $T_{a}=600 \mathrm{~K}, \varepsilon_{0}=0.124$, $N=6$ получаем $\varepsilon_{\max }=0.3 \mathrm{eV}$, что также согласуется с экспериментальными данными [16], где получено совпадение точной и восстановленной ФРИ при $\varepsilon=0.1 \mathrm{eV}$ и расхождения порядка $20 \%$ при $\varepsilon=0.5 \mathrm{eV}$ для $\mu=1$; для $\mathrm{Ar}^{+}$в $\mathrm{Ar}$ при $T_{a}=450 \mathrm{~K}, \varepsilon_{0}=0.689, N=6$ имеем $\varepsilon_{\max }=0.41 \mathrm{eV}$, что не противоречит данным [16], где для $\varepsilon=0.3 \mathrm{eV}$ получено расхождение порядка 7-8\% (напомним, что мы считали максимально допустимым расхождение в $10 \%$ ).

Как мы видели, несмотря на то, что вторая производная зондового тока при углах $\alpha=\alpha_{i}$ определяется без систематической ошибки (см. систему уравнений (2)), вычисленная из зондовых измерений анизотропная ФР

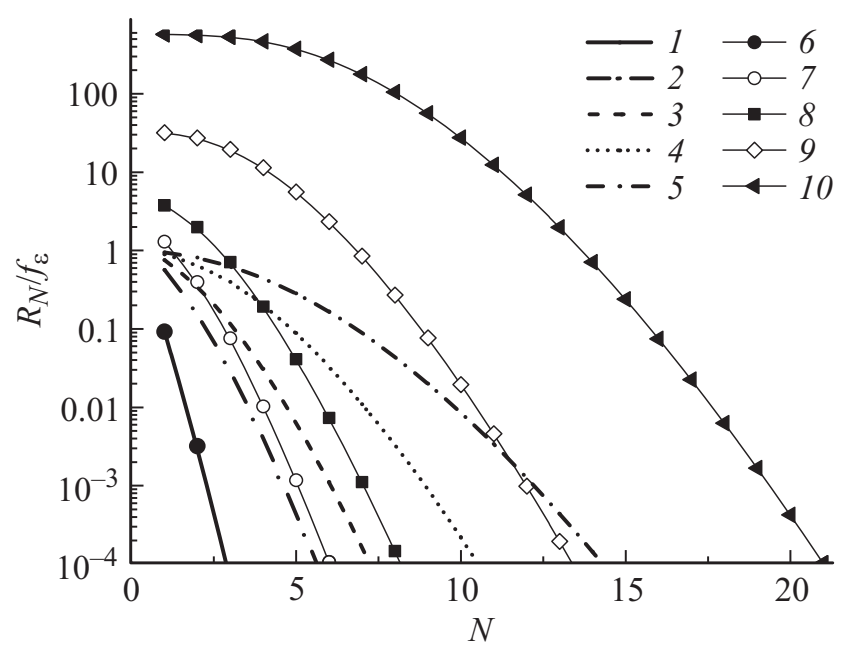

Рис. 3. Зависимость относительной ошибки при аппроксимации $Ф \mathrm{P} R_{N}(E, 0) / f_{\varepsilon}(E, 0)$ конечным рядом по полиномам Лежандра, рассчитанная для модельной ФР (определена формулами (19)) для различного числа членов ряда $N$ при $\alpha=0$ и различной анизотропии $\Phi \mathrm{P} ; E=0.1 \mathrm{eV}, \Delta_{T} E=1 \mathrm{eV}$; $1-5$ - расчет по точной формуле (7); 6-10- оценка сверху по формуле (8); $1,6-\beta=10,2,7-1 ; 3,8-4,9-0.2$, $10-0.1$.

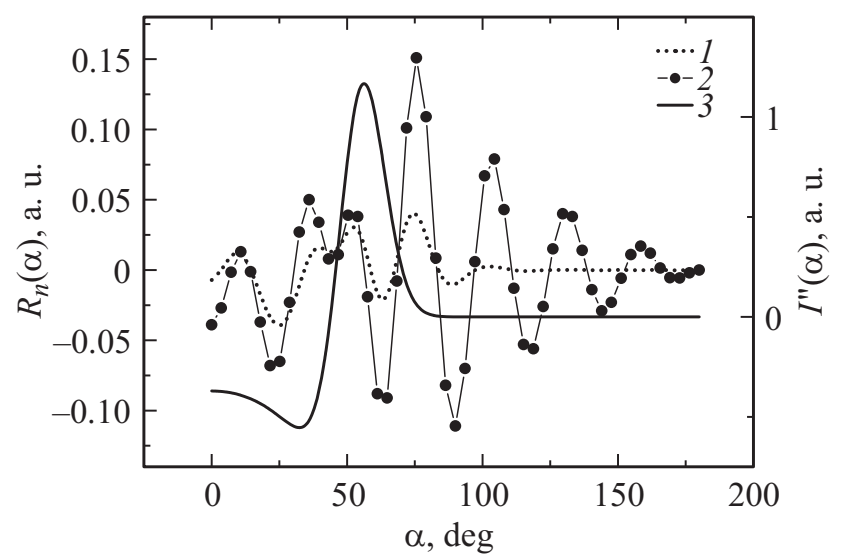

Рис. 4. Разница между точной величиной $I^{\prime \prime}(e U, \alpha)$ и вторыми производными зондового тока, вычисленной в единицах коэффициента $A$ (формула (1)) в виде ряда по 14 полиномам Лежандра и в виде ряда по 25 полиномам Лежандра с интерполяцией сплайнами по 14 точкам при $\Delta_{T} E=1 \mathrm{eV}$; $e U=0.1 \mathrm{eV} ; \beta_{1}=0.02 \mathrm{c}$ наложенной случайной ошибкой величиной $10 \% ; 1-$ результат применения сплайнов (ось $Y$ - слева), 2 - расчет в виде ряда по 14 полиномам Лежандра (ось $Y-$ слева), $3-I^{\prime \prime}(e U, \alpha)$, рассчитанная по формуле (1) (ось $Y$ - справа).

при этих углах имеет систематическую ошибку (формула (13a)). Основная физическая причина этого том, что в интегральный член в соотношении (1) дают вклад частицы, двигающиеся не по нормали к поверхности зонда (т.е. не под углами $\alpha=\alpha_{i}$ к выделенному направлению), поэтому нет однозначной связи между величинами $I^{\prime \prime}\left(e U, \alpha_{i}\right)$ и $\bar{f}_{\varepsilon}\left(E, \cos \alpha_{i}\right)$. Исключение со- 
ставляет случай, когда интегральный член в формуле (1) пренебрежимо мал, например, в ситуации, когда ФР имеет узкое по энергиям распределение.

На рис. 3 сравниваются относительные ошибки $R_{N}(E, 0) / f_{\varepsilon}(E, 0)$ представления ФР в виде конечного ряда по полиномам Лежандра для модельной функции, определенной соотношениями (19) для различного числа членов ряда $N$ при нулевом угле $\alpha$ и различной анизотропии ФР. Расчет проводился по точной формуле (7) и по формуле для оценки сверху (9). Как и следовало ожидать, при увеличении анизотропии число членов, необходимое для адекватного представления ФР в виде конечного ряда растет. При этом видно, что при малой анизотропии оценка сверху близка к точному значению относительной ошибки, в то время как при сильной анизотропии она существенно завышает необходимое количество членов. Так, если точный расчет для случая, когда $\beta_{1}=0.1$, дает необходимое число членов $N=7$, то оценка сверху дает $N=16$.

На рис. 4 приведены результаты расчетов отклонения от точной величины (рассчитанной по формулам (1), (19)) угловой зависимости величины $I^{\prime \prime}(e U, \alpha)$ в виде конечного ряда по 14 полиномам Лежандра, коэффициенты которого найдены из рассчитанных по модельной ФР при 14 разных углах $\alpha$ с наложенной ошибкой в $10 \%$. Видно, что это отклонение для представления в виде конечного ряда по 14 полиномам Лежандра существенно превышает случайную ошибку, в то время как отличия результатов разложения сплайновой интерполяции по 25 полиномам от точных значений $I^{\prime \prime}(e U, \alpha)$, напротив, ее не превосходят. Это, как отмечалось, вызвано тем, что в данном случае 14 членов ряда по полиномам Лежандра недостаточно для описания второй производной тока, формируемой сильно анизотропной ФР при $\beta=0.02$.

На рис. 5-7 сравниваются зависимости от потенциала зонда 14 коэффициентов ряда по полиномам Лежандра, полученные из значений второй производной $I^{\prime \prime}(e U, \alpha)$ для условий рис. 4 двумя исследуемыми способами. Видно, что оба набора коэффициентов примерно с одинаковой точностью описывают точные значения. Отметим, что коэффициенты $D_{2}^{14}(e U)$ (рис. 5), $D_{12}^{14}(e U)$ (рис. 6), рассчитанные с применением сплайновой интерполяции, существенно лучше описывают точные значения, a $D_{10}^{14}(e U)$ (рис. 6) - напротив, хуже, чем вычисленный традиционным способом. Несомненным преимуществом метода сплайновой интерполяции в данном случае является то, что при примерно одинаковой точности вычисления первых 14 коэффициентов Лежандра (по сравнению с традиционным способом) мы можем вычислить произвольное их число и, таким образом, повысить точность нахождения ФР. Это иллюстрируется данными рис. 8, на котором представлена разница между точным расчетом по формулам (19) и ФР, восстановленными традиционным способом в виде ряда по полиномам Лежандра с $N=10$ и с применением сплайнов в виде ряда с $M=17$. Коэффициенты Лежандра для ФР

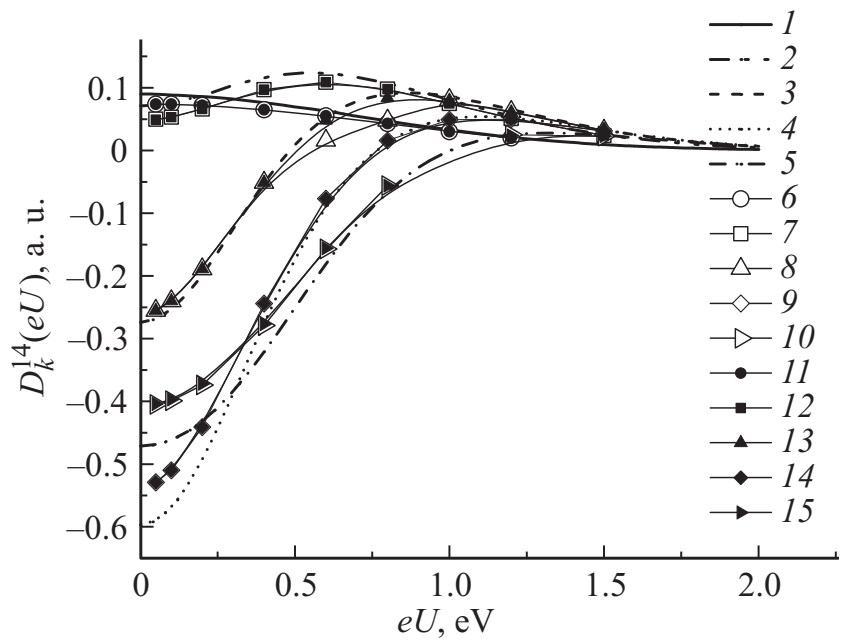

Рис. 5. Зависимость коэффициентов $D_{k}^{14}(e U)$ (при $\left.k=0-4\right)$ разложения второй производной зондового тока $I^{\prime \prime}(e U, \alpha)$ по полиномам Лежандра от потенциала зонда, рассчитанных для модельной ФР (см. формулы (19)) при $\Delta_{T} E=1 \mathrm{eV} ; \beta=0.02$ с наложенной случайной ошибкой величиной $10 \%$; кривые $1-5$ - точный расчет по модельной ФР для $k=0-4$ соответственно, 6-10 - нахождение коэффициентов из значений второй производной при 14 ориентациях зонда (традиционный способ) для $k=0-4$ соответственно, $11-15-$ нахождение этих же коэффициентов разложением в ряд по полиномам Лежандра результатов интерполяции сплайнами $I^{\prime \prime}(e U, \alpha)$ по 14 точкам при разной ориентации зонда для $k=0-4$ соответственно.

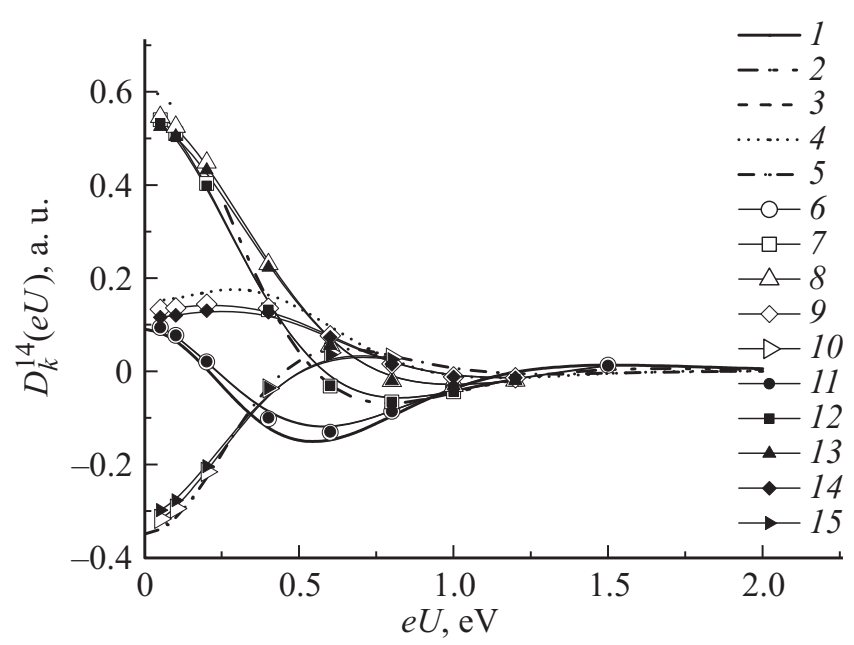

Рис. 6. То же, что на рис. 5, но для $k=5-9$; кривые $1-5-$ точный расчет $k=5-9$ соответственно, $6-10-$ нахождение коэффициентов разложения $I^{\prime \prime}(e U, \alpha)$ по полиномам Лежандра для 14 ориентаций зонда традиционным способом для $k=5-9$ соответственно, 11-15 - применение сплайновой интерполяции измерений при 14 ориентациях зонда для $k=5-9$ соответственно.

рассчитывались с использованием формулы (4). Видно, что применение сплайновой интерполяции по десяти значениям второй производной зондового тока и ис- 


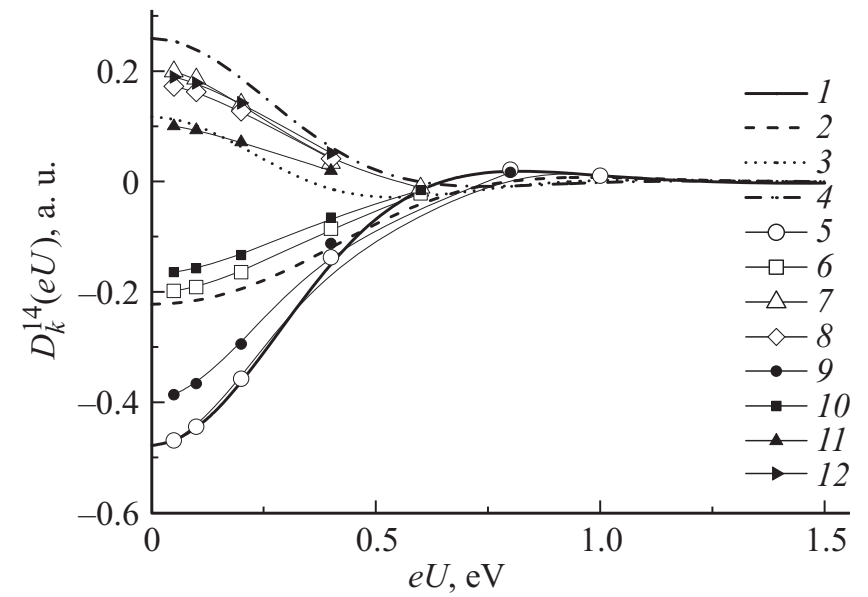

Рис. 7. То же, что и на рис. 5 , но для $k=10-14$; кривые $1-5-$ точный расчет $k=10-14$ соответственно, $6-10-$ нахождение коэффициентов разложения $I^{\prime \prime}(e U, \alpha)$ по полиномам Лежандра для 14 ориентаций зонда традиционным способом для $k=10-14$ соответственно, $11-15-$ применение сплайновой интерполяции измерений при 14 ориентациях зонда для $k=10-14$ соответственно.

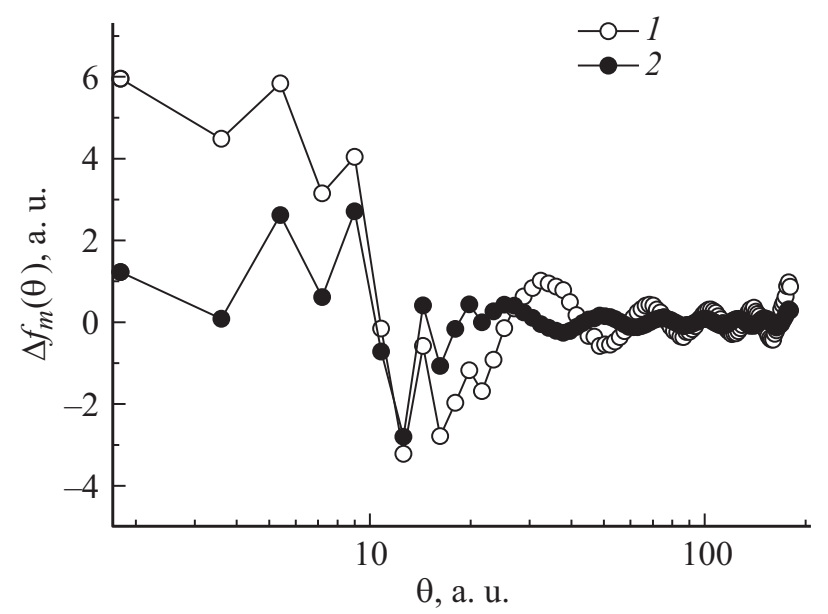

Рис. 8. Разность между точной ФР и восстановленной двумя различными способами для $\beta=0.03, \Delta_{T}=2 \mathrm{eV}, E_{0}=0$, $E=0.3 \mathrm{eV} ; 1-$ восстановленная ФР в виде суммы 10 членов ряда по полиномам Лежандра, коэффициенты которого находятся с использованием десяти значений $I^{\prime \prime}(e U, \alpha) ; 2-$ ФР восстановлена в виде суммы 17 членов ряда по полиномам Лежандра, коэффициенты которого находятся с использованием кубических сплайнов.

пользование 17 полиномов Лежандра дает существенно меньшую погрешность при $\beta=0.03$, чем традиционный метод, который в данном конкретном примере позволяет найти лишь 10 первых коэффициентов ряда (3).

\section{Заключение}

В работе исследованы основные причины расхождения экспериментально определенных методом плоского одностороннего зонда анизотропных ФРЭ и ФРИ с их точными значениями.

Для условий, когда анизотропия вызвана электрическим полем в плазме, показано, что в большом числе практически важных случаев (исключая приэлектродные слои, где величина электрического поля может быть аномально большой) для точного описания ФРИ достаточно провести измерения при 10 и менее (в зависимости от степени анизотропии ФРИ) ориентациях плоского зонда относительно выделенного направления.

Что касается применения сплайнов для уменьшения ошибки данного метода измерения анизотропных ФР, то использование вышеописанного алгоритма целесообразно в основном в ситуациях, когда степень анизотропии слишком велика и практически реализуемого числа ориентаций зонда недостаточно для точного описания ФР в виде конечного ряда по полиномам Лежандра. Если же экспериментальная установка позволяет при зондовых измерениях реализовать достаточно высокое угловое разрешение, то применение сплайновой интерполяции не дает заметного повышения точности по сравнению с традиционным способом определения коэффициентов разложения ФР.

Отметим также, что учет зеркальной симметрии во многих задачах исследования ФР заряженных частиц в плазме позволяет при том же числе ориентаций зонда практически в два раза увеличить число членов ряда по полиномам Лежандра по сравнению со случаем отсутствия симметрии, или для фиксированного числа членов ряда настолько же сократить необходимое число ориентаций зонда.

\section{Приложение 1}

Формулы для величины $\varepsilon_{\max }\left(N, \varepsilon_{0}, 1\right)$ в диапазоне изменения параметра $0.01 \leq \varepsilon_{0} \leq 1.5$ разбиты на четыре поддиапазона:

1) $0.01 \leq \varepsilon_{0} \leq \mathbf{0 . 2}$

$\varepsilon_{\max }\left(N, \varepsilon_{0}, T_{a} 1\right)=F_{01}\left(N, T_{a}\right)+F_{11}\left(N, T_{a}\right) \varepsilon_{0}+F_{21}\left(N, T_{a}\right) \varepsilon_{0}^{2}$; $F_{01}\left(N, T_{a}\right)=a_{01}^{(1)} N+a_{02}^{(1)} N^{2} ; F_{11}\left(N, T_{a}\right)=a_{11}^{(1)} N+a_{12}^{(1)} N^{2}$; $F_{21}\left(N, T_{a}\right)=a_{21}^{(1)} N+a_{22}^{(1)} N^{2}$;

$a_{01}^{(1)}=g_{010}^{(1)}+g_{011}^{(1)} T_{a}+g_{012}^{(1)} T_{a}^{2}$;

$a_{02}^{(1)}=g_{020}^{(1)}+g_{021}^{(1)} T_{a}+g_{022}^{(1)} T_{a}^{2}$;

$a_{12}^{(1)}=g_{120}^{(1)}+g_{121}^{(1)} T_{a}+g_{122}^{(1)} T_{a}^{(2)}$;

$a_{21}^{(1)}=g_{210}^{(1)}+g_{211}^{(1)} T_{a}+g_{212}^{(1)} T_{a}^{2}$;

$a_{22}^{(1)}=g_{221}^{(1)} T_{a}$;

$g_{010}^{(1)}=-0.01 ; g_{011}^{(1)}=1.1 \cdot 10^{-4} ; g_{012}^{(1)}=-6.67 \cdot 10^{-8}$;

$g_{020}^{(1)}=0.017 ; g_{021}^{(1)}=-3.83 \cdot 10^{-5} ; g_{022}^{(1)}=5.56 \cdot 10^{-8}$;

$g_{120}^{(1)}=-6 \cdot 10^{-4} ; g_{121}^{(1)}=2.42 \cdot 10^{-6} ; g_{122}^{(1)}=1.39 \cdot 10^{-9}$;

$g_{210}^{(1)}=-3 \cdot 10^{-3} ; g_{211}^{(1)}=3.5 \cdot 10^{-5} ; g_{212}^{(1)}=-1.67 \cdot 10^{-8}$

2) $0.2 \leq \varepsilon_{0} \leq \mathbf{0 . 7}$

$\varepsilon_{\max }\left(N, \varepsilon_{0}, T_{a} 1\right)=F_{02}\left(N, T_{a}\right)+F_{12}\left(N, T_{a}\right) \varepsilon_{0}+F_{22}\left(N, T_{a}\right) \varepsilon_{0}^{2}$; $F_{02}\left(N, T_{a}\right)=a_{00}^{(2)} N+a_{02}^{(2)} N^{2} ; F_{12}\left(N, T_{a}\right)=a_{13}^{(2)} N^{3}$; 
$F_{22}\left(N, T_{a}\right)=a_{22}^{(2)} N^{2}$

$a_{00}^{(2)}=g_{000}^{(2)}+g_{001}^{(2)} T_{a}+g_{002}^{(2)} T_{a}^{(2)}$

$a_{02}^{(2)}=g_{020}^{(2)}+g_{021}^{(2)} T_{a}+g_{022}^{(2)} T_{a}^{2}$

$a_{13}^{(2)}=g_{130}^{(2)}+g_{131}^{(2)} T_{a}+g_{132}^{(2)} T_{a}^{(2)}$

$a_{22}^{(2)}=g_{220}^{(2)}+g_{211}^{(2)} T_{a}+g_{222}^{(2)} T_{a}^{2}$;

$g_{000}^{(2)}=-3 \cdot 10^{-3} ; g_{011}^{(2)}=2.5 \cdot 10^{-5} ; g_{002}^{(2)}=-5.56 \cdot 10^{-9}$;

$g_{020}^{(2)}=5 \cdot 10^{-4} ; g_{021}^{(2)}=1.55 \cdot 10^{-5} ; g_{022}^{(2)}=-5.56 \cdot 10^{-10}$;

$g_{130}^{(2)}=3 \cdot 10^{-4} ; g_{131}^{(2)}=-3.83 \cdot 10^{-6} ; g_{132}^{(2)}=1.67 \cdot 10^{-9}$;

$g_{220}^{(2)}=-3 \cdot 10^{-4} ; g_{221}^{(2)}=5.83 \cdot 10^{-5} ; g_{222}^{(2)}=-2.78 \cdot 10^{-8}$

3) $0.7 \leq \varepsilon_{0} \leq \mathbf{1}$;

$\varepsilon_{\max }\left(N, \varepsilon_{0}, T_{a} 1\right)=F_{03}\left(N, T_{a}\right)+F_{13}\left(N, T_{a}\right) \varepsilon_{0}$;

$F_{03}\left(N, T_{a}\right)=a_{00}^{(3)} N+a_{01}^{(3)} \frac{\{\ln (N-2)\}^{3}}{N^{1.1}} ;$

$F_{13}\left(N, T_{a}\right)=a_{11}^{(3)} \frac{\{\ln N\}^{3}}{N^{0.7}}$;

$a_{00}^{(3)}=g_{000}^{(3)}+g_{001}^{(3)} T_{a}+g_{002}^{(3)} T_{a}^{2}$;

$a_{01}^{(3)}=g_{010}^{(3)}+g_{011}^{(3)} T_{a}+g_{012}^{(3)} T_{a}^{2}$;

$a_{11}^{(3)}=g_{110}^{(3)}+g_{111}^{(3)} T_{a}+g_{112}^{(3)} T_{a}^{2}$;

$g_{000}^{(3)}=-0.222 ; g_{001}^{(3)}=9 \cdot 10^{-5} ; g_{002}^{(3)}=-5.56 \cdot 10^{-8}$;

$g_{010}^{(3)}=0.25 ; g_{011}^{(3)}=-4.42 \cdot 10^{-3} ; g_{012}^{(3)}=2.5 \cdot 10^{-6}$;

$g_{110}^{(3)}=-0.22 ; g_{111}^{(3)}=4.18 \cdot 10^{-3} ; g_{112}^{(3)}=-2.78 \cdot 10^{-6}$;

4) $1 \leq \varepsilon_{0} \leq \mathbf{1 . 5}$

$\varepsilon_{\max }\left(N, \varepsilon_{0}, T_{a} 1\right)=F_{04}\left(N, T_{a}\right)+F_{14}\left(N, T_{a}\right) \varepsilon_{0}$;

$F_{04}\left(N, T_{a}\right)=a_{00}^{(4)} N+a_{01}^{(4)} N^{2}$

$F_{14}\left(N, T_{a}\right)=a_{10}^{(4)}+a_{11}^{(4)} N+a_{12}^{(4)} N^{2}$;

$a_{00}^{(4)}=g_{000}^{(4)}+g_{001}^{(4)} T_{a}+g_{002}^{(4)} T_{a}^{2}$;

$a_{01}^{(4)}=g_{010}^{(4)}+g_{011}^{(4)} T_{a}+g_{012}^{(4)} T_{a}^{2}$;

$a_{11}^{(4)}=g_{110}^{(4)}+g_{111}^{(4)} T_{a}+g_{112}^{(4)} T_{a}^{2}$;

$a_{12}^{(4)}=g_{120}^{(4)}+g_{121}^{(4)} T_{a}+g_{122}^{(4)} T_{a}^{2}$;

$g_{000}^{(4)}=-9 \cdot 10^{-3} ; g_{001}^{(4)}=-2.45 \cdot 10^{-4} ; g_{002}^{(4)}=3.11 \cdot 10^{-6}$;

$g_{010}^{(4)}=0.379 ; g_{011}^{(4)}=1.29 \cdot 10^{-3} ; g_{012}^{(4)}=-4.45 \cdot 10^{-6}$;

$g_{020}^{(4)}=-0.376 ; g_{021}^{(4)}=-9.48 \cdot 10^{-4} ; g_{022}^{(4)}=1.31 \cdot 10^{-6}$;

$g_{100}^{(4)}=0.017 ; g_{101}^{(4)}=2.13 \cdot 10^{-4} ; g_{102}^{(4)}=-3.08 \cdot 10^{-6}$;

$g_{110}^{(4)}=-0.395 ; g_{111}^{(4)}=-1.2 \cdot 10^{-3} ; g_{122}^{(4)}=4.4 \cdot 10^{-6}$;

$g_{120}^{(4)}=0.378 ; g_{121}^{(4)}=9.88 \cdot 10^{-4} ; g_{122}^{(4)}=-1.33 \cdot 10^{-6}$;

Расчеты произведены с точностью порядка $10 \%$.

\section{Список литературы}

[1] Fedorov V.L. // Zh. Tekh. Fiz. 1985. Vol. 55. P. 926. [Sov. Phys. Tech. Phys. 1985. Vol. 30. P. 554.]

[2] Mezentsev A.P., Mustafaev A.S., Fedorov V.L. // Zh. Tekh. Fiz. 1985. Vol. 55. P. 544. [Sov. Phys. Tech. Phys. 1985. Vol. 30. P. 322.]

[3] Mezentsev A.P., Mustafaev A.S. // Zh. Tekh. Fiz. 1985. Vol. 55. P. 2232. [Sov. Phys. Tech. Fiz. 1985. Vol. 30. P. 1319.]

[4] Mezentsev A.P., Mustafaev A.S., Lapshin V.F., Fedorov V.L. // Zh. Tekh. Fiz. 1986. Vol. 58. P. 2104. [Sov. Phys. Tech. Phys. 1986. Vol. 31. P. 1263.]

[5] Mustafaev A.S., Mezentsev A.P. // J. Phys. D. 1986. Vol. 19. L69.

[6] Mezentsev A.P., Mustafaev A.S., Lapshin V.F., Fedorov V.L. // J. Phys. B. 1987. Vol. 20. L723.
[7] Mezentsev A.P., Mustafaev A.S., Fedorov V.L. // J. Phys. D. 1988. Vol. 21. P. 1464.

[8] Fedorov V.L., Mezentsev A.P. // Zh. Tekh. Fiz. 1987. Vol. 57. P. 595. [Sov. Phys. Tech. Phys. 1987. Vol. 32. P. 363.$]$

[9] Lapshin V.F., Mustafaev A.S. // Zh. Tekh. Fiz. 1989. Vol. 59. P. 35. [Sov. Phys. Tech. Phys. 1989. Vol. 34. P. 150.]

[10] Klagge S., Lunk A. // J. Appl. Phys. 1991. Vol. 70. P. 99.

[11] Klagge S. // Plasma Chem. Plasma Process. 1992. Vol. 12. P. 103.

[12] Mal'kov M.A. // Fiz. Plazmy. 1990. Vol. 16. P. 467. [Sov. J. Plasma Phys. 1990. 1B. P. 267.]

[13] Woods R.C., Sudit I.D. // Phys. Rev. E. 1994. Vol. 50. N 3. P. 2222-2238.

[14] Mustafaev A.S., Sukhomlinov V.S., Ainov M.A. // Techn. Phys. 2015. Vol. 60. N 12. P. 1778-1789.

[15] Мустафаев А.С., Сухомлинов В.С., Аинов М.А. // ТВТ. 2016. принята к печати.

[16] Мустафаев А.С., Сухомлинов В.С., Аинов М.А. // ТВТ. 2016. принята к печати.

[17] Langmuir I., Mott-Smith H.M. // Phys. Rev. 1926. Vol. 28. P. 723.

[18] Лебедев Ю.А. Электрические зонды в плазме пониженного давления. http:// plasma.karelia.ru?pub/fntp/Lebedev.pdf

[19] Смирнов Б.М. Ионы и возбужденные атомы в плазме. М.: Атомиздат, 1974. $456 \mathrm{c.}$

[20] Абрамовии М., Стиган И. Справочник по специальным функциям с формулами, графиками и математическими таблицами. М.: Наука, 1979. 831 с.

[21] Федорюк М.В. Метод перевала. М.: Наука, 1977. 368 с.

[22] Де Бор $К$. Практическое руководство по сплайнам. М.: Радио и связь, 1985. 304 с.

[23] Soukhomlinov V., Mustafaev A., Strahova A., Kaganovich I. // Abstract Submitted for the $44^{\text {th }}$ IEEE International Conference on Plasma Science, ICOPS-2017. 2017. Atlantic City, NJ, USA.

[24] Мустафбаев А.С. // ЖТФ. 2001. Т. 71. № 4. C. 111-121.

[25] Мустабаев А.С. Функция распределения электронов в анизотропной плазме. СПб.: Национальный минеральносырьевой университет „Горный“, 2013. 172 с. 